\title{
Hormonal control of calcium ingestion: The effects of neonatal manipulations of the gonadal steroids both during and after critical stages in development on the calcium ingestion of male and female rats
}

\author{
JOHN J. REILLY and JAY SCHULKIN \\ University of Pennsylvania, Philadelphia, Pennsylvania
}

\begin{abstract}
Female rats ingest greater amounts of calcium than do their male counterparts. We inquired into the endocrine regulation of this phenomenon. We replicated our earlier findings that virgin mature females ingest more calcium than males do. We also found that neonatal gonadectomy ( 1 day) during critical stages in development, but not after ( 30 days), resulted in female rats' decreasing their calcium ingestion when tested when mature. We found that neonatal gonadectomy in males resulted in increased calcium ingestion behavior. Taken in conjunction with our earlier results, which showed that adult gonadectomy (60 days) had no effect on calcium ingestion, these results demonstrate that neonatal circulation of gonadal steroids induce organizational changes in the brain to alter calcium ingestion.
\end{abstract}

Female rats are known to ingest greater amounts of sodium than do males; this is true of virgins (Krecek, Novakova, \& Stribrad, 1972), but is even more conspicuous during pregnancy and lactation in a number of species (Denton, 1982; Richter, 1956; Schulkin, 1991b). This greater avidity for sodium is determined by neonatal hormonally induced changes in the brain, which help to determine subsequent behavior (Chow, Sakai, Witcher, Adler, \& Epstein, 1992; Krecek, 1973, 1978). This behavioral expression is biologically important. We reasoned that, like sodium ingestion, a similar phenomenon might hold for calcium ingestion.

Our earlier work has demonstrated a relationship between the severity of the calcium deprivation and the amount of calcium ingested (Tordoff, Ulrich, \& Schulkin, 1990). Other inquirers have shown that calcium-deprived rats, pigs, chickens, and monkeys (see reviews by Richter, 1943, 1956; Rozin \& Schulkin, 1990) increased their calcium intake. Moreover, like sodium intake, calcium intake increases during pregnancy and lactation (Denton, 1982; Richter, 1943; Woodside \& Millelire, 1986).

Female virgin rats also express a greater avidity for calcium than do males. We showed in an earlier study that adult gonadectomy did not affect the calcium ingestion in male and female rats (Schulkin, 1991a). In the following two experiments, we inquired into the effects of ma-

We thank Micah Leshem, Bruce McEwen, and Paul Rozin for their comments on the text, and Stella Chow for technical advice for the gonadectomies. The research was supported by NIMH grants. Correspondence should be addressed to J. Schulkin, Behavioral Neuroscience Unit, Clinical Neuroendocrinology Branch, National Institute of Mental Health, Bldg. 10, Rm. 3N228A, 9000 Rockville Pike, Bethesda, MD 20892. nipulating the gonadal steroid hormones during the neonatal period to determine whether this would alter calcium ingestion in adulthood.

\section{EXPERIMENT 1}

In the first experiment, we asked whether or not neonatal gonadectomy affects the ingestion of calcium in mature female and male rats.

\section{Method}

Twenty Sprague-Dawley rats (two litters of rats with 5 male and 5 female rats in each litter) at 1 day of age were gonadectomized. Half of the litter at a time was removed from the mother. Each pup was anesthetized using hypothermia. The gonads were surgically removed as previously described (e.g., Chow et al., 1992). Briefly, for female gonadectomy, two dorsal cuts caudal to the ribs were made on each side, first through the skin and then through the layer of muscle. Following the removal of the gonads, the muscle and the skin were sutured. For the male gonadectomy, two ventral cuts just lateral to the penis were made on each side, first through the skin then through the muscle. Following the removal of the gonads, the muscle and the skin were sutured. After suturing, the pups were placed on an isothermal heating pad to speed the return to normal body temperature. After the pups returned to normal behavior (movements, exploration), they were cleaned and returned to the mother; the other half of the litter was then removed for surgery. A control litter ( 5 males and 5 females) was removed from the dam for this same period and anesthetized, but without the surgery.

At 23 days of age, the pups were weaned and were group-housed in a temperature-controlled room, under a 12:12-h light:dark cycle. At 60 days of age, they were individually housed and given access to $2.4 \%$ calcium lactate (made fresh daily), in addition to ad-lib water and Purina chow (calcium-rich diet). The ingestion of calcium was measured every $24 \mathrm{~h}$ over a 10-day period. The 24-h ingestion was averaged over the 10-day period 
The results are presented as means. Appropriate analyses of variance (ANOVAs) were performed on all data, and significance level was set at $p<.05$.

\section{Results and Discussion}

The results replicate our earlier findings that females ingest more calcium than do males (Figure $1 ; p<.01$ ). Figure 1 shows that neonatal gonadectomy in females reduced their calcium ingestion dramatically, relative to gonadally intact controls $(p<.05)$. Figure 1 also shows that neonatal gonadectomy in males tended to increase calcium ingestion compared to that of the gonadally intact male $(p<.05)$. The ingestion of calcium in gonadectomized male and female rats was not different (Figure 1).

Figure 2 reveals that the gonadally intact male rat's body weight is significantly higher than that of each of the other three groups $(p<.01)$. This result replicates the well-known facts that male rats are heavier than fe-

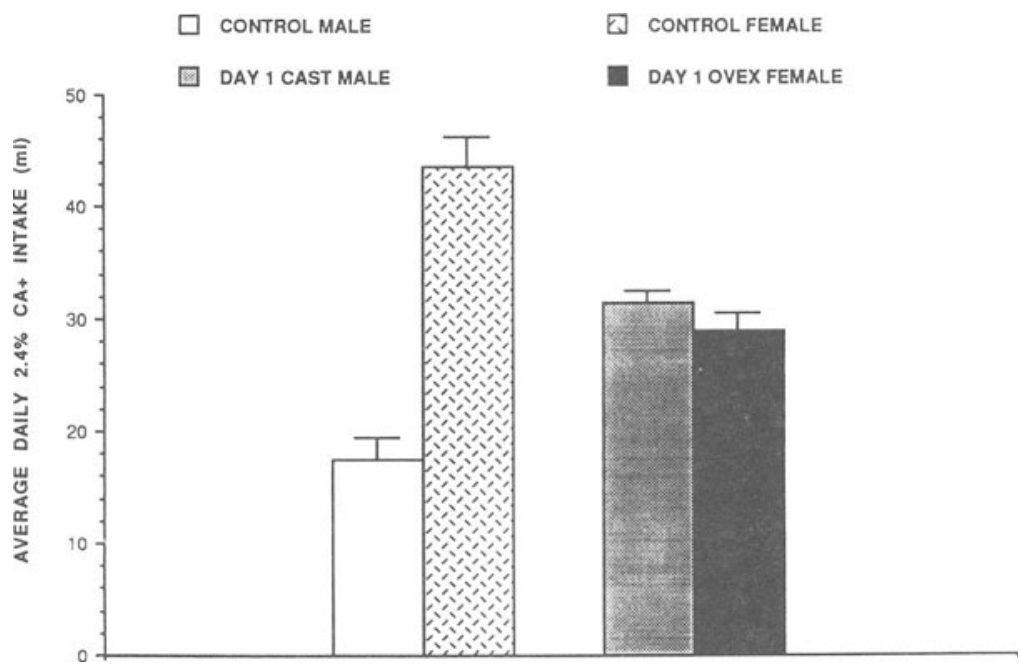

Figure 1. The 24-h calcium intake (Ms and $S D$ s) of control male and female rats and gonadectomized (1 day) male and female rats.

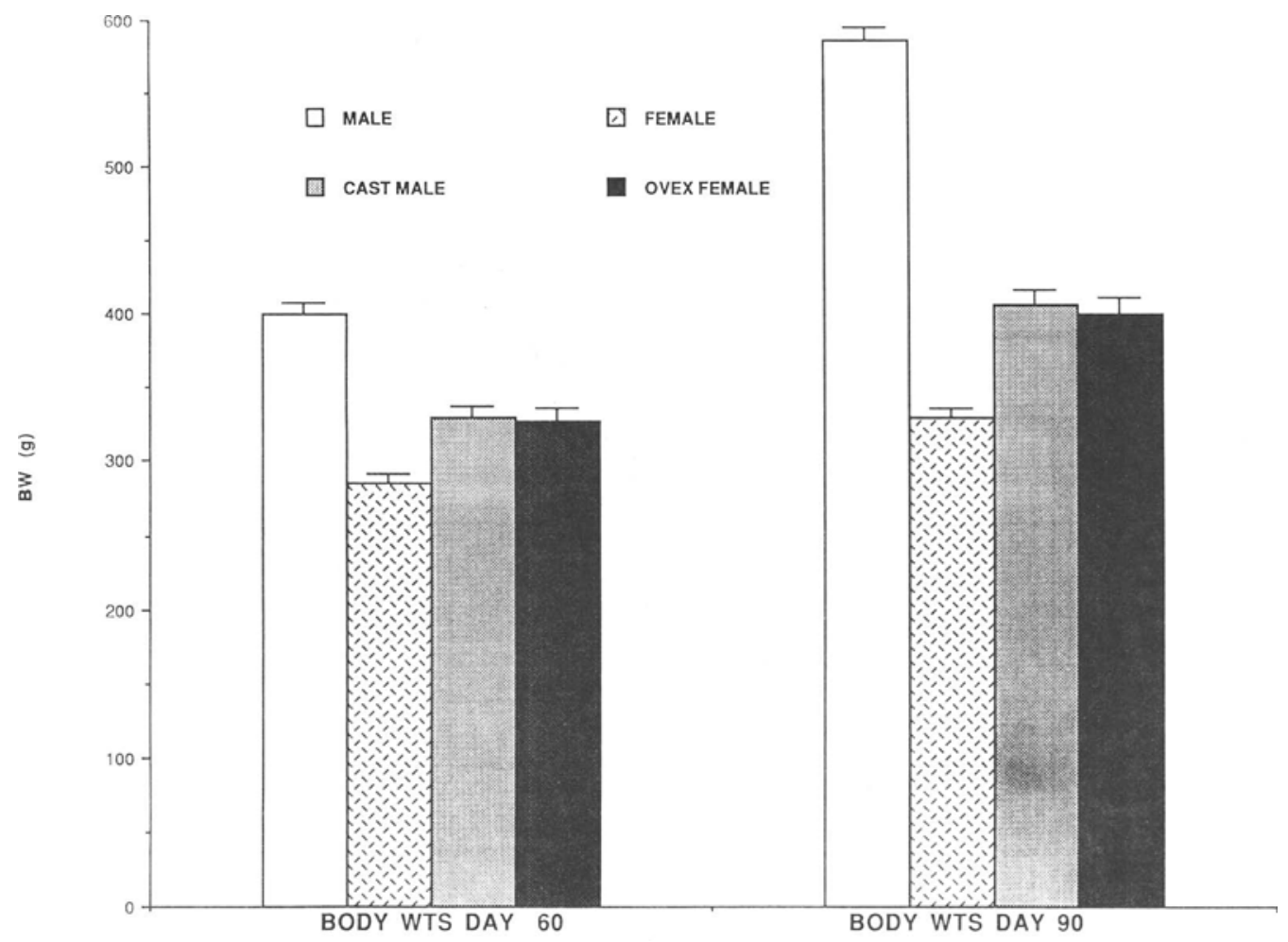

Figure 2. The body weights (Ms and SDs) of the male and female rats in Experiment 1. 
male rats and that gonadectomized male rats tend to have body weights comparable to that of female rats (Chow et al., 1992; see review by Goy \& McEwen, 1980).

These results suggest that neonatal gonadectomy alters the calcium intake of mature male and female rats.

\section{EXPERIMENT 2}

In Experiment 2, we extended the results of Experiment 1 , by adding several new groups and by looking at the ingestion of different concentrations of the calcium lactate solution.

\section{Method}

Ten male and 10 female rats were gonadectomized at either 1 day of age (as described in Experiment 1) or 30 days of age, and
10 male and 10 female rats served as sham-operated controls. We also gave female rat testosterone (TP, Sigma T1875) via a Silastic capsule placed subcutaneously in the back, and it remained in throughout the experiment (as described in Chow et al., 1992). Each 30-day-old rat was anesthetized using an intramuscular injection of a Ketamine-Acepromazine cocktail (10:1.6 by volume; $0.7 \mathrm{ml} / \mathrm{kg}$ BW) and given $0.1 \mathrm{ml}$ of the antibiotic Gentamycin. The gonads were then removed (see Chow et al., 1992, for more details). After suturing, the animal was placed on an isothermal heating pad to speed the return to normal body temperature (movements and righting behavior), after which it was returned to its cage.

The rats were tested as described in Experiment 1. However, in Experiment 2, they were first given the $2.4 \%$ calcium lactate for 2 weeks, followed by $3.6 \%$ or $1.2 \%$ for 1 week. We used different concentrations of calcium to determine whether the basic effect held for both more and less palatable solutions than that used in Experiment 1 . Again, calcium solutions were made fresh daily, and tubes were cleaned daily to avoid precipitation. Ingestion was again moni-

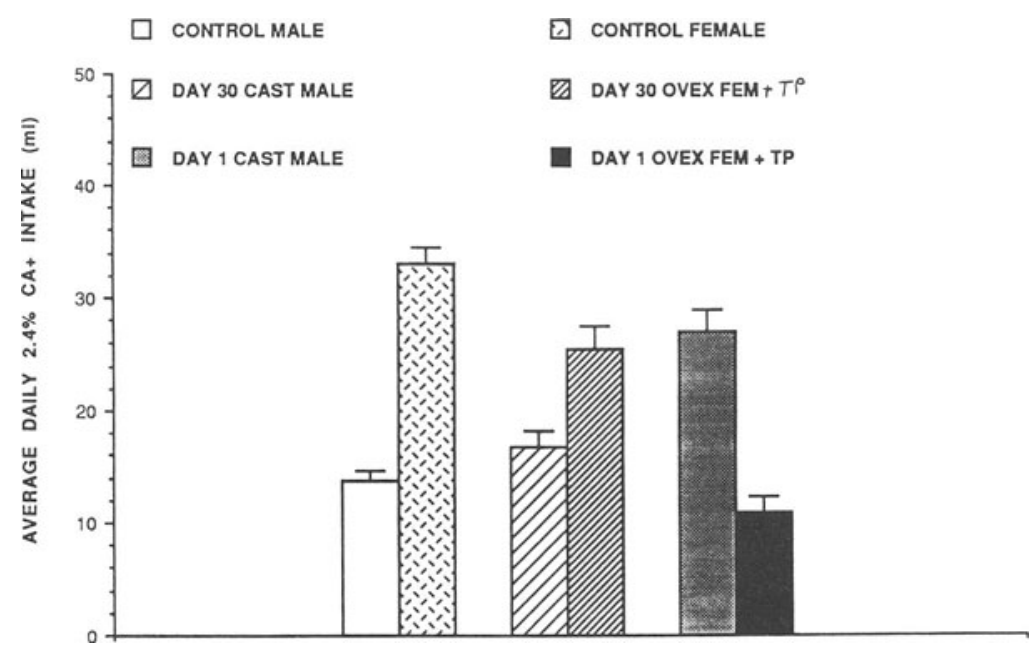

Figure 3. The 24-h $2.4 \%$ calcium intake ( $M$ s and $S D$ s) of control male and female rats, gonadectomized rats treated with testosterone at 1 day of age or 30 days of age, and gonadectomized male rats treated at 1 day or 30 days of age.

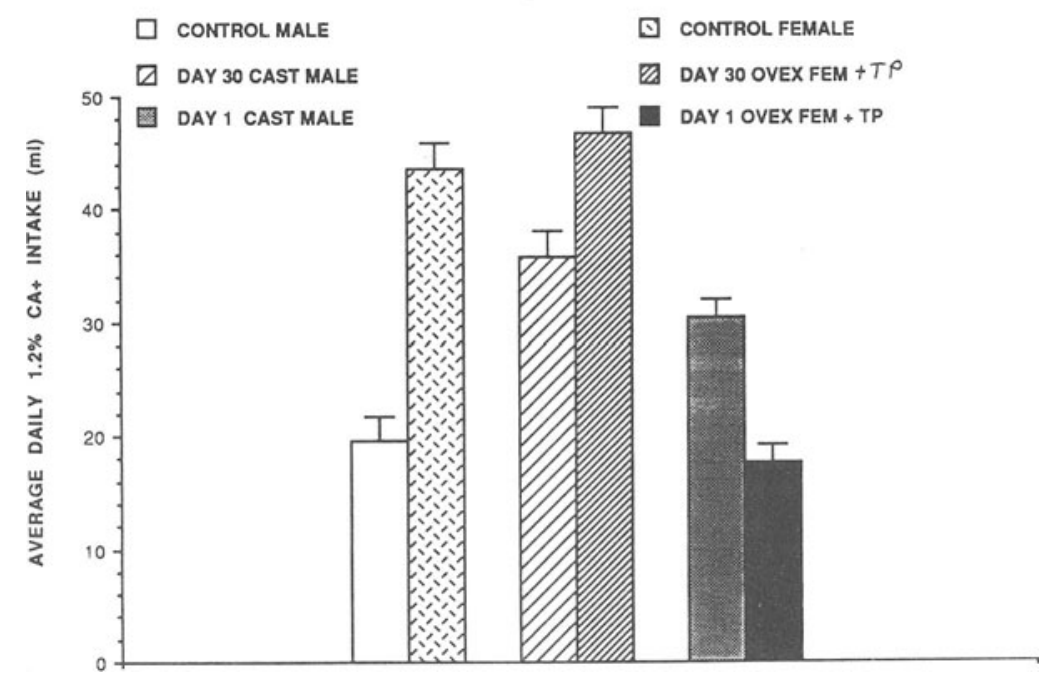

Figure 4. The 24h $1.2 \%$ calcium intake (Ms and SDs) of control male and female rats, gonadectomized rats treated with testosterone at 1 day of age or 30 days of age, and gonadectomized rats treated at 1 day or 30 days of age. 
tored every $24 \mathrm{~h}$, and food and water were given ad lib in addition to the calcium. Ingestion was averaged over this time period.

\section{Results and Discussion}

Again, we found that control female rats ingest more calcium than do their male counterparts $(p<.01)$; this held for all concentrations of calcium (Figures 3,4 , and 5 ).

Gonadectomized female rats, at 1 day of age, but not at 30 days of age, ingested less calcium, relative to that ingested by control females at all concentrations $(p<.01$; Figures 3, 4, and 5).

Gonadectomized male rats, at 1 day of age, but not at 30 days, increased their calcium ingestion, relative to that of intact control males at the 2.4 and $3.6 \%$ concentrations ( $p<.01$; Figures 3 and 5 ). However, both groups demonstrated elevated ingestion of the calcium at the $1.2 \%$ concentration (Figure 4), relative to controls $(p<.01)$.

Gonadectomized female rats at 1 day of age ingested less calcium than did their male counterparts at all three concentrations ( $p<.01$; Figures 3,4 , and 5). But the same trend of elevated calcium ingestion in females, relative to that in males, held for the gonadectomized rats at 30 days of age ( $p<.01$; Figures 3,4 , and 5).

Figure 6 reveals that the gonadally intact male rat's body weight is significantly higher than that of each of the other groups at 90 days of age $(p<.01)$, but not at 60 days of age $(p>.01)$; however, the males gonadectomized at $\mathbf{3 0}$ days were significantly different from control males $(p<.01)$.

These results therefore confirm the observations of Experiment 1 . They show that calcium ingestion is a sexually dimorphic behavior. When taken with our previous report (Schulkin, 1991a) that gonadectomy at 60 days of age did not affect calcium ingestion in male and female rats, these results show that gonadal steroid hormones de- termine calcium ingestion during critical stages in neonatal development.

\section{CONCLUSION}

Our results show that neonatal gonadectomy has effects on calcium ingestion in adult male and female rats. For females, it decreases calcium ingestion; for males, it increases calcium intake. When taken together with earlier results, which showed that adult gonadectomy has no effect on calcium ingestion (Schulkin, 199la) and that 30-day-old manipulations were generally not effective in altering calcium ingestion, these results demonstrate that neonatal gonadal steroid action on the brain has organizational effects on calcium ingestion. Thus, like sexual behavior (Goy \& McEwen, 1980), in addition to sodium (Chow et al., 1992; Krecek, 1978) or saccharin ingestion (Zucker, 1969), the actions of the gonadal steroid hormones during critical stages have long-term consequences for behavior.

What we do not know from the present results are the mechanisms of action. It is well known that testosterone metabolized to estrogen results in defeminization of the brain with its influence on sexual and physiological functions (Goy \& McEwen, 1980). Our results show that female gonadectomy with or without testosterone treatment results in decreased calcium ingestion. Future research will determine the mechanisms for the changes in calcium ingestion.

In closing, consider the biological context for calcium ingestion. It is during pregnancy and lactation that calcium (in addition to other minerals) is ingested in even greater amounts than normal (Denton, 1982; Richter, 1956). It is during reproduction when calcium is needed

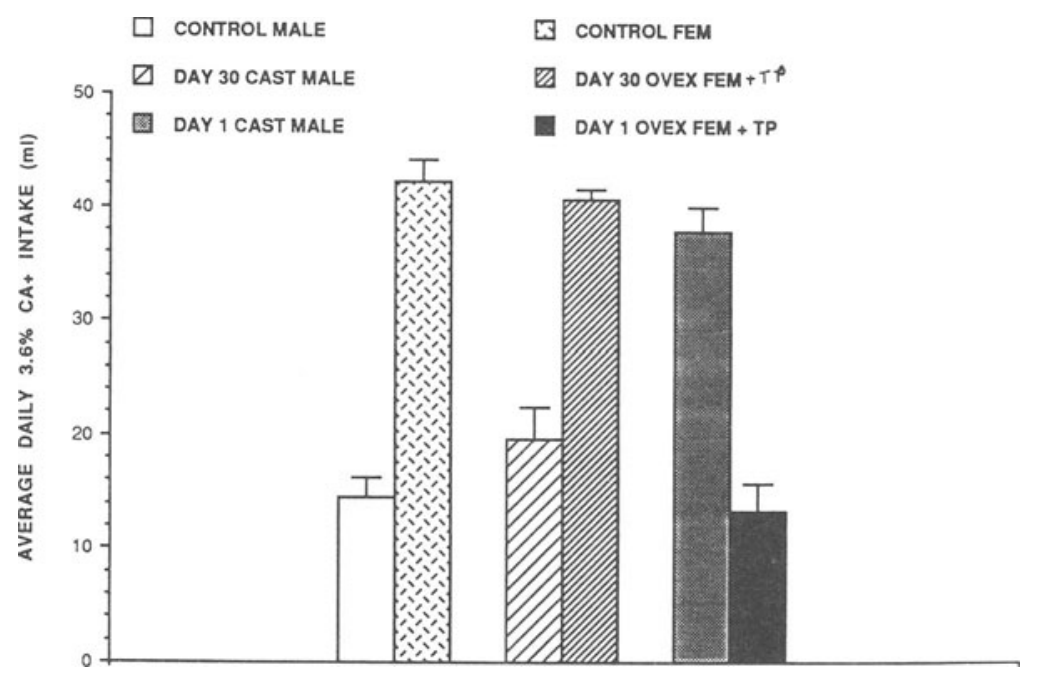

Figure 5. The 24-h 3.6\% calcium intake ( $M$ s and $S D s$ ) of control male and female rats, gonadectomized rats treated with testosterone at 1 day of age of 30 days of age, and gonadectomized rats treated at 1 day or 30 days of age. 


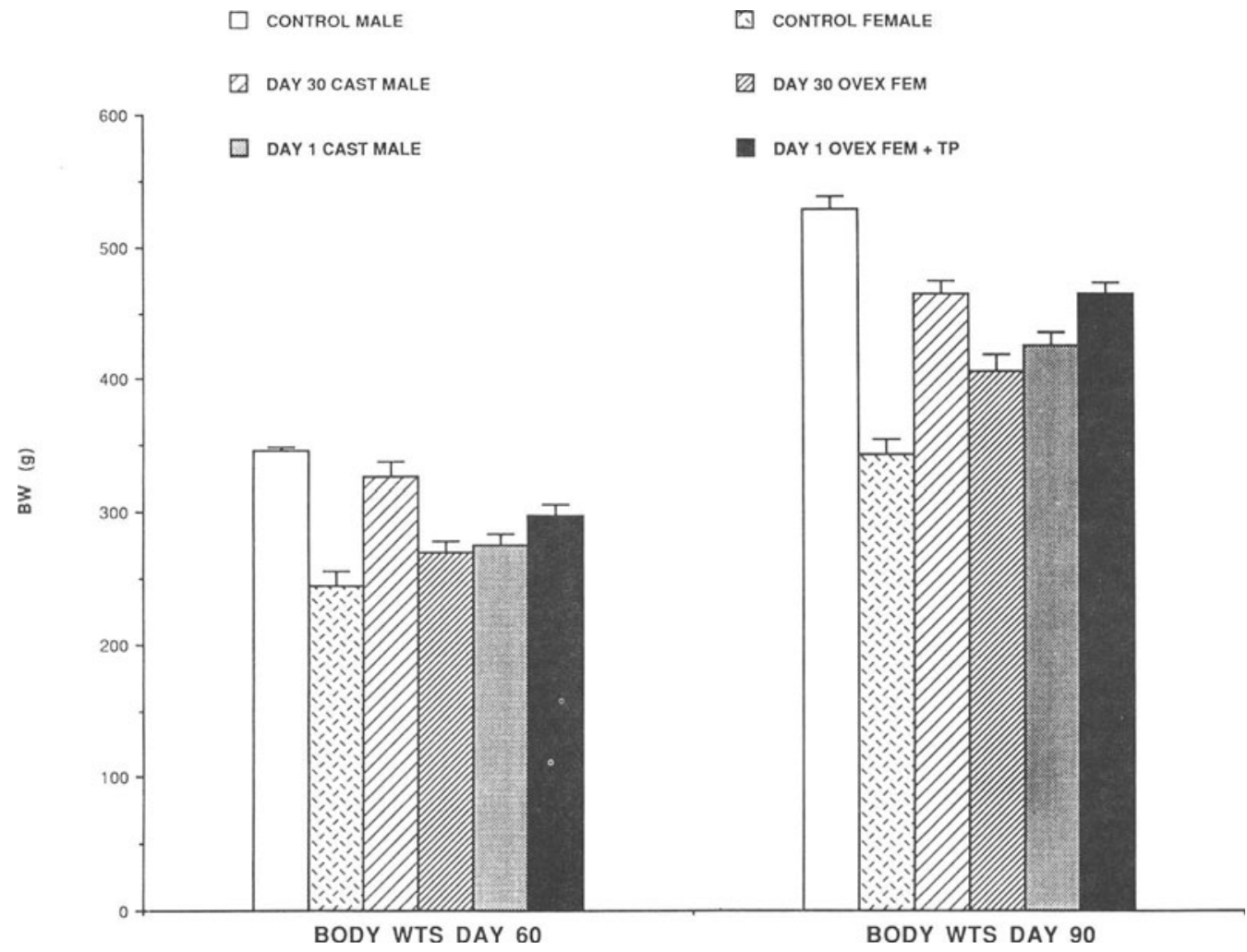

Figure 6. The body weights (Ms and SDs) of the male and female rats in Experiment 2.

by the female. Evolution therefore ensured adequate mineral homeostasis in female rats by enhancing the avidity for calcium during this time. But there are also increases in calcium ingestion when rats are deprived of calcium (e.g., Tordoff et al., 1980). Moreover, as we have shown, there is also an enhanced ingestion of calcium (Schulkin, 1991b) when female rats (relative to male rats) are not in need of calcium, and neonatal hormonal events have long-term consequences on calcium ingestion.

Elsewhere, we have suggested that sexually dimorphic brain sites that are induced by neonatal actions of the gonadal steroid hormones may underlie the sexually dimorphic expression of mineral ingestion (Schulkin, 1991b). Perhaps, the same sexually dimorphic sites that are involved in sodium hunger (medial nucleus of the amygdala) and that are involved in other sexually differentiated behaviors (see review by Schulkin, 1991b) are also involved in the enhanced avidity for calcium in female rats.

\section{REFERENCES}

Chow, S., Sakai, R. R., Witcher, J. A., Adler, N. T., \& Epstein, A. N. (1992). Sex and sodium intake in the rat. Behavioral Neuroscience, 106, 172-180.

DENTON, D. (1982). The hunger for salt. New York: Springer-Verlag. GoY, R. W., \& MCEwEN, B. S. (1980). Sexual differentiation of the brain. Cambridge, MA: MIT Press.
KrECEK, J. (1973). Sex differences in salt taste: The effect of testosterone. Physiology \& Behavior, 10, 683-688.

KRECEK, J. (1978). Effect of ovariectomy of females and estrogen administration to males during the neonatal critical period on salt intake in adulthood in rats. Physiologia Bohemoslovaca, 27, 1-5.

Krecex, J., Novakova, V., \& Stribrad, K. (1972). Sex differences in the taste preference for a salt solution in the rat. Physiology \& Behavior, 10, 183-188.

Richter, C. P. (1943). Total self regulatory functions in animals and human beings. Harvey Lecture Series, 38, 63-105.

RICHTER, C. P. (1956). Salt appetite of mammals: Its dependence on instinct and metabolism. In Linstinct dans le comportement des animaux et de lhomme (pp. 577-629). Paris: Masson.

Rozin, P., \& Schulkin, J. (1990). Food selection. In E. Stricker (Ed.), The handbook of behavioral neurobiology (pp. 297-328). New York: Plenum.

SCHULKIN, J. (1991a). The ingestion of calcium in female and male rats. Psychobiology, 19, 262-264.

SchULkin, J. (1991b). Sodium hunger: The search for a salty taste. Cambridge: Cambridge University Press.

TordofF, M. J., Ulrich, P. M., \& SChulkin, J. (1990). Calcium deprivation increases salt intake. American Journal of Physiology, 28 , R411-R419.

Woodside, B., \& MiLleliRe, L. (1986). Self-selection of calcium during pregnancy and lactation in rats. Physiology \& Behavior, 39, 291-285.

ZUCKER, I. (1969). Hormonal determinants of sex differences in saccharin preference, food intake and body weight. Physiology \& Behavior, 4, 595-602.

(Manuscript received June 23, 1992; revision accepted for publication September 24, 1992.) 